\title{
Forest Structure Characterization From SAR Tomography at L-Band
}

\author{
Marivi Tello ${ }^{\circledR}$, Member, IEEE, Victor Cazcarra-Bes ${ }^{\circledR}$, Matteo Pardini ${ }^{\circledR}$, Member, IEEE, \\ and Konstantinos Papathanassiou $\left.{ }^{(}\right)$, Fellow, IEEE
}

\begin{abstract}
Synthetic aperture radar (SAR) remote sensing configurations are able to provide continuous measurements on global scales sensitive to the vertical structure of forests with a high spatial and temporal resolution. Furthermore, the development of tomographic SAR techniques allows the reconstruction of the threedimensional (3-D) radar reflectivity opening the door for 3-D forest monitoring. However, the link between 3-D radar reflectivity and 3-D forest structure is not yet established. In this sense, this paper introduced a framework that allows a qualitative and quantitative interpretation of physical forest structure from tomographic SAR data at L-band. For this, forest structure is parameterized into a set of a horizontal and a vertical structure index. From inventory data, both indices can be derived from the spatial distribution and the dimensions of the trees. Similarly, two structure indices are derived from the 3-D spatial distribution of the local maxima of the reconstructed 3-D radar reflectivity profiles at L-band. The proposed methodology is tested by means of experimental tomographic L-band data acquired over the temperate forest site of Traunstein in Germany. The obtained horizontal and vertical structure indices are validated against the corresponding estimates obtained from inventory measurements and against the same indices derived from the vertical profiles of airborne Lidar data. The high correlation between the forest structure indices obtained from these three different data sources (expressed by correlation coefficients between 0.75 and 0.87 ) indicates the potential of the proposed framework.
\end{abstract}

Index Terms-Forest structure, synthetic aperture radar, tomography.

\section{INTRODUCTION}

$\mathbf{F}$ OREST structure is eminently linked to the threedimensional (3-D) size, location, and arrangement of trees, trunks, and branches in a forest [1], [2] and reflects therefore the forest state and its evolution [3]-[5]. Accordingly, forest structure is an indicator of forest successional stage and development as well as sustainability and habitability and is therefore an important parameter for assessing forest productivity [6], biomass level, and biodiversity [7]-[9]. Forest structure changes reveal dynamic processes as growth, regeneration, decay, and natural or anthropogenic disturbance. Knowledge about such processes

Manuscript received November 7, 2017; revised March 20, 2018 and June 29, 2018; accepted July 8, 2018. Plot establishment and the first survey for this work were supported by the Helmholtz Research Alliance "Remote Sensing and Earth System Dynamics" and in part by the Initiative and Networking Fund of the Helmholtz Association. (Corresponding author: Victor Cazcarra-Bes.)

The authors are with the German Aerospace Center (DLR), Microwaves and Radar Institute (HR), Wessling 82234, Germany (e-mail: victor.cazcarrabes@ dlr.de; matteo.pardini@dlr.de; kostas.papathanassiou@dlr.de).

Color versions of one or more of the figures in this paper are available online at http://ieeexplore.ieee.org.

Digital Object Identifier 10.1109/JSTARS.2018.2859050 is important for modeling the function and the evolution of forest ecosystems and for developing accurate and robust forest biomass estimators [10].

The wide range of applications that require forest structure information imposes a wide range of measurement and observation requirements. For biomass and carbon applications, yearly global scale monitoring at spatial resolutions on the order of 1 ha are sufficient [3]. On the other hand, ecology and biodiversity applications are often addressed at landscape-to-regional level and require forest structure information at spatial and temporal scales associated to the occurring changes, i.e., down to single tree level with monthly to seasonal updates [7].

These requirements cannot be satisfied by the currently available means of forest monitoring. Traditionally, forest structure characterization relies on sampling at local scales by means of field inventory plots or more recently terrestrial laser scanning techniques able to catch the 3-D arrangement of vegetation compartments. However, any extrapolation to larger scales depends on the ability of these measurements to represent their surrounding landscape. At the same time, in many cases, the temporal continuity of such plots measurements is very difficult to be established.

Remote sensing techniques have the potential to overcomeat least some of-these limitations and to make a significant contribution in qualitative and quantitative monitoring of 3-D forest structure [3], [7]-[10]. Today, two remote sensing technologies allow the measurement of 3-D forest information: waveform Lidar and synthetic aperture radar (SAR) tomography. While the capabilities and challenges of the different Lidar configurations for mapping 3-D forest structure are today well established and widely understood, the potential of tomographic SAR configurations is not fully assessed yet. Note here that the horizontal variability alone that can be estimated by conventional remote sensing techniques is often not sufficient to describe unambiguously vegetation structure [10] so that 3$\mathrm{D}$ descriptors and measurements are required for a complete characterization.

SAR tomography relies on a set of SAR images acquired under slightly different incidence angles as for example along slightly displaced tracks or orbits to estimate the 3-D distribution of the backscattered power, also known as the 3-D radar reflectivity. The reconstruction the 3-D radar reflectivity is today established and has been demonstrated in several experiments across different forest ecosystems [11]-[15]. The Appendix summarizes the main tomographic reconstruction algorithms. 
However, the link between the reconstructed 3-D radar reflectivity and the physical forest structure is not as well understood and far from being established. There are several reasons that make this task challenging. One of them is related to the interpretation of the reconstructed 3-D radar reflectivity in terms of 3-D forest structure attributes. Obviously, the 3-D radar reflectivity depends on the system frequency and polarization employed, on the acquisition geometry (e.g., incidence angle) used, and on the (3-D) spatial resolution achieved. The fact that the scatterers "seen" by the radar change with frequency and polarization makes a generic interpretation of the 3-D reflectivity difficult. In addition, with decreasing frequency, the scattering contribution of the ground under the forest adds to the one of the forest making the interpretation of lower forest components located close to the ground more difficult. This paper focuses on 3-D radar reflectivity at L-band, where in general a significant penetration into and through the forest volume is expected implying scattering contributions from the forest volume and from the underlying ground.

While forest structure is primarily associated to the geometric properties of tree and forest stand elements, the radar reflectivity depends in addition to geometric properties also on the dielectric properties of the scattering elements of the forest. This makes the interpretation of the reconstructed 3-D radar reflectivityand especially its change-ambiguous. As a consequence, the estimation of forest structure has to be robust enough to reflectivity variations that are not relevant to structure as for example caused by rain or temperature gradients [12], [13] while still remaining sensitive enough to morphological variations.

When attempting to relate the reconstructed 3-D radar reflectivity to physical forest structure descriptors established in forestry and/or ecology, one faces a significant discrepancy. The physical forest structure descriptors are built on individual tree parameters that do not have a direct correspondence in the reconstructed 3-D radar reflectivity due to the insufficient spatial resolution of the SAR configurations that does not allow individualizing scattering contributions of single trees. This paper aims to contribute to the interpretation of L-band 3-D radar reflectivity in terms of physical forest structure. In this sense, a framework that allows the estimation of qualitative and quantitative 3-D forest structure information from 3-D radar reflectivity reconstructed by SAR tomography at L-band is proposed. In Section II, forest structure estimation from field and remote sensing data is discussed and a pair of structure indices that allow the characterization of horizontal and vertical forest structure is introduced. The two indices allow setting up a schema for the systematic characterization and quantification of 3-D forest structure that can be applied on inventory and remote sensing data. In Section III, experimental results obtained from airborne tomographic SAR data acquired at L-band and (airborne) Lidar data over the temperate forest of Traunstein in Germany are presented. The structure indices derived from the tomographic SAR, the Lidar, and the inventory measurements are discussed and compared with each other. The advantage of 3-D forest structure information versus forest height information only is discussed. The effect of the spatial scale on the interpretation of the obtained results is also assessed. Finally, conclusions are drawn in Section IV.

\section{Forest StruCture Estimation}

\section{A. Forest Structure Estimation From Field Data}

Since both forestry and ecology have been traditionally based on field data, the discussion on forest structure metrics has been essentially carried out on an individual tree basis. On plot level, it is usually approached by estimating different attributes based on individual trees within the plot, such as height, basal area, canopy dimensions, species composition, and/or stand density, which are then used to derive a structure index. Numerous indices have been proposed in the literature, such as the aggregation index [16], the diametric differentiation index, the mingling index, the contagion index, or the complex stand index [17]. However, all of them have limitations in the sense that no one provides a univocal characterization of any possible tree distribution within the stand [18]-[21]. As a consequence, there is not yet an overall measure able to express forest structure in terms of forestry or ecology. Nevertheless, despite the difficulties in defining an index appropriate for a wide span of applications and forest types, there is a common understanding that in order to express forest structure, two complementary aspects of forest structure need to be considered, namely the structural heterogeneity in the horizontal and in the vertical dimension. In this sense, horizontal structure reflects stand density, whereas vertical structure accounts for tree size variability.

Regarding horizontal structure, it is worth noting here that stand density is not exclusively related to the number of trees per unit area, but rather to site occupancy and therefore tree volume per area [5]. One standard metric in forest practice is absolute basal area [22]. But in order to compare stands of different ages, it is preferable to introduce measures linking space utilization to tree size [21]. The most common among them is the stand density index [23], closely related to the basal area. It relates the stand density with the equivalent density of a stand with an actual quadratic mean diameter of $25 \mathrm{~cm}$. In the scope of this paper, this metric is employed for the estimation of horizontal structure from field data:

$$
\mathrm{HS}_{\text {field }}=N\left(\frac{D_{g}}{25}\right)^{1.605}
$$

expressed in trees per hectare, where $N$ is the number of stems per hectare and $D_{g}$ is the quadratic mean diameter at breast height in $\mathrm{cm}$ [24], within the structure window. In the following, the area of the structure window, i.e., the size of the polygon enclosing the trees that are accounted when computing the statistical quantities defined in (1)-(4) will be referred to as unit scale. Several subsequent refinements of this index have been discussed in the literature [25]. Accounting only for the tallest trees in the stand allows a better characterization of canopy closure, while being more consistent by assuming the same allometric relationship between the size and density for all the trees involved in the estimation. Also, without loss of generality, $\mathrm{HS}_{\text {field }}$ is normalized to its maximum and then $1-\mathrm{HS}_{\text {field norm }}$ 


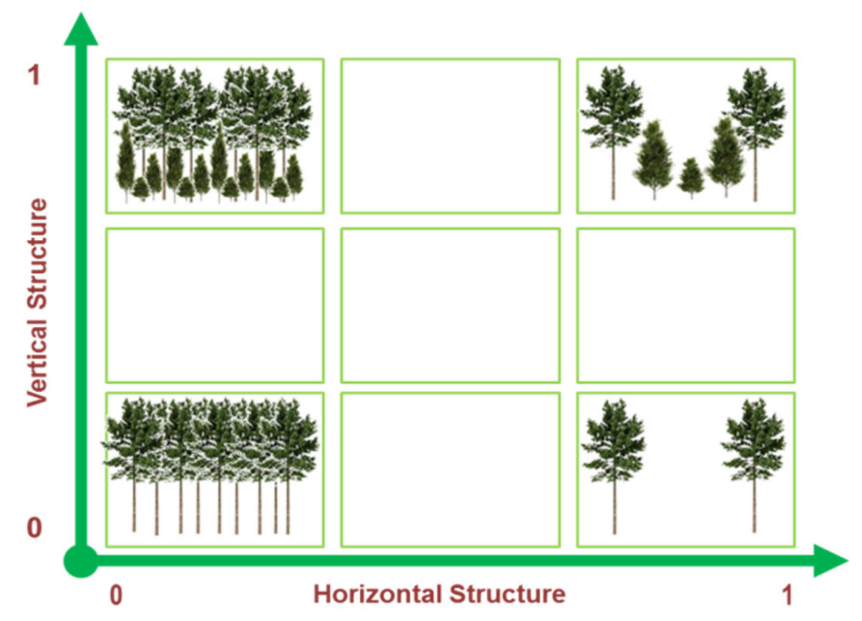

Fig. 1. Horizontal and vertical forest structure measures are complementary and allow the definition of a plane where stands can be projected according to their structure complexity. As an example, the position of four different examples of forest stand is shown here.

is employed in order to reflect disorder: 0 indicates low and 1 indicates high structural disorder or complexity.

Vertical structure can be evaluated by the size differentiation, the Gini coefficient, the Shannon index [26], or as the standard deviation of tree heights [6], [27], among others. However, due to the fact that tree height measurements are often not performed, these indices are usually not possible to be estimated directly from inventory data. As an alternative, the standard deviation of tree diameter at breast height (dbh) that reflects as well the tree size variability [5] has been used that can be directly estimated from standard inventory measurements. Furthermore, according to [2] and [4], dbh variability appears to be more sensitive to successional stages than measures of height diversity alone. Therefore, in the scope of this paper, vertical forest structure from field data $\mathrm{VS}_{\text {field }}$ is estimated as the standard deviation of tree dbh:

$$
\mathrm{VS}_{\text {field }}=\operatorname{std}(\{\mathrm{dbh}\})
$$

expressed in $\mathrm{cm}$, where $\{\mathrm{dbh}\}$ is the ensemble of diameter at breast height of all the trees included in the structure window, given in $\mathrm{cm}$. Without loss of generality, the vertical forest structure descriptor from field data is normalized to its maximum within the image and becomes unitless.

Using now the horizontal $\mathrm{HS}_{\text {field }}$ and vertical $\mathrm{VS}_{\text {field }}$ structure indices, it is possible to define a plane, referred to as $\mathrm{HV}$ plane in the following, on which forest stands can be projected depending on their structure (see Fig. 1). The complementary aspect of horizontal and vertical structure can be observed in Fig. 1. Four stands are projected in the plane defined by their horizontal and vertical structure. The four stands sketched can only be unambiguously discriminated when both the horizontal and the vertical dimensions are accounted for. First, two monolayered stands have been sketched. They have low vertical structure because they are mainly constituted by trees of similar size. The difference between them relies in the horizontal axis, due to their different density. On the other hand, two multilayered
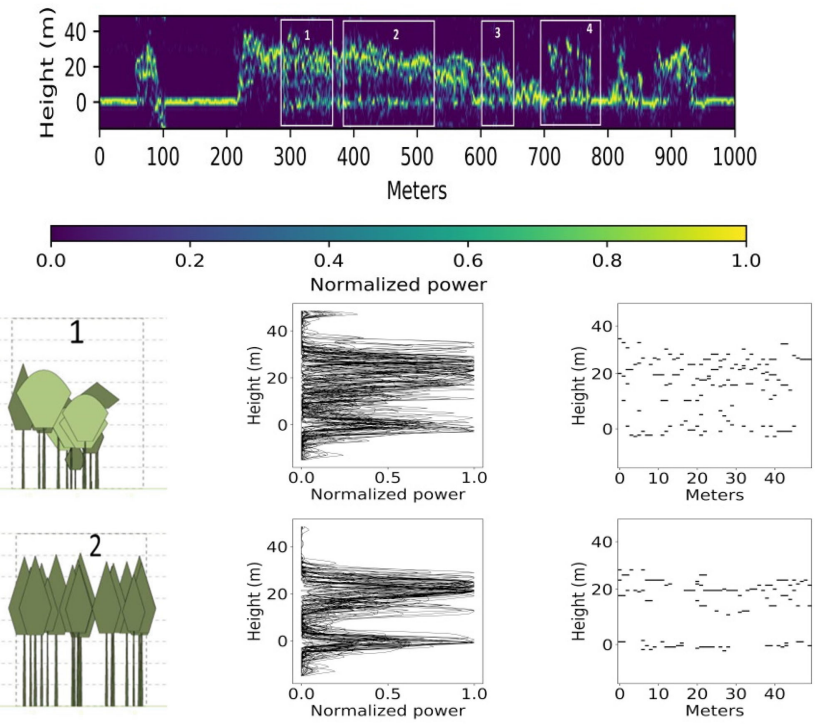

3
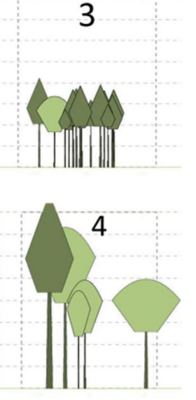
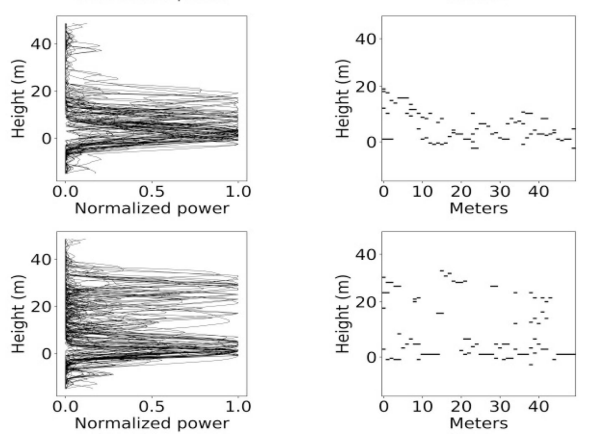

Fig. 2. (Top row) Example of normalized tomogram along a managed highly heterogeneous temperate forest. (Left column) Schematic representation of the trees distributions, (middle column) corresponding reflectivity profiles superimposed, and (right column) peaks of the reflectivity profiles, for the four areas highlighted in the tomogram.

stands have been considered as well. They both have similar high vertical structure. The difference between them relies again in the horizontal dimension.

\section{B. Forest Structure Estimation From TomoSAR Data}

The objective of this section is to define a framework for the estimation of forest structure from L-band TomoSAR data. For this, the definition of structure indices applicable to TomoSAR data that meet the same notions as the forest structure indices derived from inventory data discussed in the previous section will be attempted. The challenge is to circumvent the fact that individual tree-based measures are usually not possible to be retrieved with TomoSAR data, which hampers a direct translation of the metrics employed in ecology and forestry. The main principles and processing steps in SAR tomography relevant for this paper are summarized in the Appendix.

1) Physical Interpretation of Reflectivity Profiles: Fig. 2 shows an example of an (normalized) L-band 3-D reflectivity transect (referred as tomogram in the following) through a managed temperate forest, crossing stands at different growth stages. Four different areas are highlighted and denoted by numbers. For each of these four areas, a representation of the tree 
distribution as obtained from the field inventory data is given (left column) and the corresponding reflectivity profiles (middle column). Looking at the two representations makes evident that the differences in the 3-D reflectivity profiles reflect the differences in forest structure for each of the areas. As indicated by the schematic tree representations, areas 2 and 3 are monolayered dense stands, whereas areas 1 and 4 are constituted by significantly more diverse tree sizes. Looking at the reflectivity profiles, besides the ground, most of the maxima are concentrated in a $10 \mathrm{~m}$ height range going from 20 to $30 \mathrm{~m}$ and from 10 to $20 \mathrm{~m}$ for areas 2 and 3, respectively, whereas they are distributed along more than $30 \mathrm{~m}$, from 10 to $40 \mathrm{~m}$ for both areas 1 and 4 . The 3-D distribution of the local reflectivity maxima (i.e., the reflectivity peaks) shown in the right column of Fig. 2 is distinctive for the four different forest structure types considered in this example.

This indicates that the 3-D distribution of reflectivity peaks at L-band reflects the variability in the distribution of trees. Even if the physical significance of reflectivity peaks in terms of forest structure is not established, a number of models and experiments in the literature support this assumption [28]-[30]. From an experimental perspective, the comparison of vertical reflectivity profiles at L-band with the height distribution of trees derived from airborne laser scanning data confirms that reflectivity peaks are produced at the range of heights occupied by the branches [31].

The discussion above triggers the idea to define measures of forest structure based on the (spatial) 3-D distribution of reflectivity peaks ignoring their (absolute) intensity. This choice has several advantages. First, it allows to attenuate the impact of radar reflectivity variations due to nonstructural effects. The location of the reflectivity peaks appears significantly more robust than the relative distribution of energy against changes induced by rain events, temperature gradients, seasonality [13], and/or the choice of the TomoSAR algorithm itself [32]. This allows the use of tomographic super-resolution algorithms that allow to critically improve the vertical resolution beyond the Rayleigh resolution, by taking into account the associated loss of radiometric accuracy and or consistency. Among all TomoSAR algorithms in the literature, compressive sensing (CS) provides the highest super-resolution under the relevant TomoSAR conditions, and for this reason it has been used in the following (see the Appendix). At the same time, using the distribution of peaks only allows to reduce the dimensionality of the observation space required for the definition of a structure metric.

2) Definition of Horizontal and Vertical Forest Structure Indices From TomoSAR: The objective of this section is to define indices of horizontal and vertical structure from the 3-D spatial distribution of the ensemble of reflectivity peaks. These indices should reflect the same notions of spatial variability of the canopy cover and tree size distribution as the indices defined in (1) and (2). Following the discussion in Section II-B1, it can be assumed that the presence of forest layers at different heights within a stand is reflected by a higher variability in the height of the reflectivity peaks. Based on this, it is possible to define one horizontal and one vertical index of structure from the 3-D distribution of the reflectivity peaks.

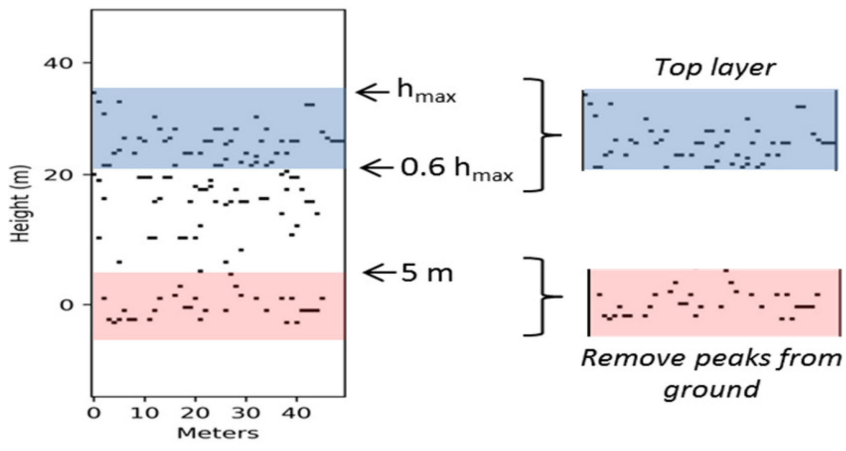

Fig. 3. Definition of top layer for the horizontal structure index and the threshold to remove peaks introduced by the ground scattering contributions.

For the horizontal structure index HS, first the height of the highest peak in the unit scale $h_{\max }$ is estimated and a top layer is defined as the range of heights between $0.6 h_{\max }$ and $h_{\max }$. The blue layer in Fig. 3 shows the top layer as obtained for the first area shown in Fig. 2. Once the top layer is defined, the horizontal structure descriptor is computed as the number of peaks within the top layer divided by the unit area $S$. Note that the definition of the top layer is rather empirical, relying though on a definition suggested in [33].

Let's assume $P=\left\{p_{1}, p_{2}, \ldots p_{O}\right\}$ the set of $O$ reflectivity peaks in a given unit scale and $H_{P}=\left\{h_{1}, h_{2}, \ldots h_{M}\right\}$ the set of $M$ heights at which these peaks are produced. With this, the horizontal structure index is defined as follows:

$$
\mathrm{HS}=\frac{n\left(P_{\mathrm{top}}\right)}{S}
$$

where $n\left(P_{\text {top }}\right)$ is the total number of peaks in the top layer.

Without loss of generality and similar to $\mathrm{HS}_{\text {field, }} \mathrm{HS}$ is normalized to its maximum $\mathrm{HS}_{\text {norm }}$ within the scene and $1-\mathrm{HS}_{\text {norm }}$ is considered in order to reflect disorder: 0 indicates low and 1 indicates high structural disorder or complexity.

The vertical structure index VS is defined as follows:

$$
\mathrm{VS}=M^{\prime} \operatorname{var}\left(H_{p}^{\prime}\right)
$$

where $H_{P}^{\prime}$ is the vector of size $M^{\prime}$ obtained from $H_{P}$ after accounting all peaks that appear at the same height only once and ignoring the peaks of the ground. At lower frequencies, the presence of the ground scattering contributions introduces reflectivity peaks that are not directly related to the forest volume. In order to exclude the ground induced peaks, a minimum height is set and peaks below this height are disregarded. In this paper, the height threshold is set to $5 \mathrm{~m}$ (see the red area in Fig. 3), implying that understory $(1-3 \mathrm{~m})$ is also ignored. Finally, the descriptor of the vertical forest structure VS is normalized to its maximum $\mathrm{VS}_{\text {norm }}$ within the scene.

Several considerations should be raised here. First, while the majority of peaks occur at the position of the canopies, this is not always the case. This can be due to sidelobes in the reconstruction of the vertical reflectivity as a result of a suboptimum sampling (i.e., number and/or distribution of acquisitions), the insufficiency of the reconstruction algorithm, or the erroneous 
detection of reflectivity peaks (see the reflectivity profiles in Fig. 2). As a consequence, it is of advantage to define forest structure descriptors by means of a statistical measure in order to be robust enough to false peak detections. In this context, no reliable information can be extracted from a single peak and the larger the number of peaks, the more accurate the estimated height frequency distribution becomes, ensuring a sufficient number of peaks impose restrictions on the range of spatial scales that can be employed. The chosen scale should be large enough to comprise a statistically meaningful number of trees and small enough to occupy a homogeneous area in terms of structure. The definition of these bounds is not straightforward, essentially because they depend on the forest heterogeneity. For instance, compared to homogeneous stands, a highly structured stand requires sampling on a larger scale to reflect its heterogeneity.

Furthermore, the structure indices introduced rely on the assumption that a group of trees of similar height causes a reflectivity peak at the height of the common canopy. However, groups of trees of the same height but different species, might produce peaks at different heights as a result of the different crown architecture (i.e., shape) introducing an ambiguity in the vertical structure index.

Finally, it should be noted that the indices suggested in this section are not absolute measures. Different systems at different resolutions might produce a different set of peaks for the same scene. Since the measure of vertical structure reflects a relative distribution of peaks in the vertical dimension, it will not be affected by differences in range and azimuth resolution. The horizontal one may present lower values with poorer resolutions. In order to account for this, before estimating the structure measures, the ensemble of peaks is projected on a geographic grid always at a resolution of $1 \times 1 \mathrm{~m}$. This step allows bringing the number of peaks identified by systems at different resolutions to comparable ranges. This framework has already proven its validity in forest structure changes tracking in a temporal series of TomoSAR data acquired by systems with different characteristics essentially in terms of resolution (range, azimuth, and vertical) and viewing geometries [34].

\section{EXPERIMENTAL RESUlTS ON A TEMPERATE FOREST}

\section{A. Description of the Test Site and Available Dataset}

In this section, the method suggested for forest structure estimation from TomoSAR data at L-band is applied on a temperate forest. The test site considered is Traunstein, a managed forest located in South-Eastern Germany, recently included in the ForestGEO network. The location, diameter at breast height and species of around 16000 trees were sampled in 2015 in an area of 25 ha (see the area indicated with a black perimeter in Fig. 4). In terms of structure, the site is characterized by a spatial structure gradient with different stages of management. The eastern part is mostly covered by monospecific homogeneous stands, while the western part is dominated by multilayered mixed stands.

In May 2017, a tomographic radar dataset at L-band was acquired over the area in a repeat pass interferometric mode by
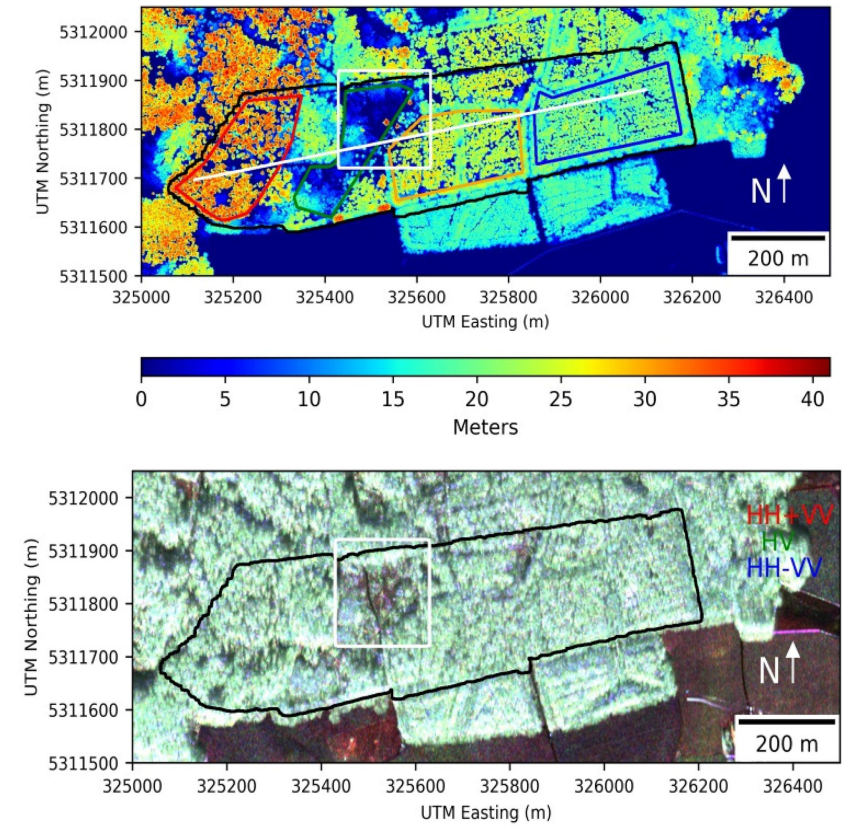

Fig. 4. (Top) Map of Lidar heights over the test site and (bottom) Pauli representation (i.e., using $|\mathrm{HH}-\mathrm{VV}|,|\mathrm{HV}|$ and $|\mathrm{HH}+\mathrm{VV}|$ as the RGB channels).

TABLE I

MAIN PARAMETERS OF THE TOMOGRAPHIC SAR DATASET

\begin{tabular}{|c|c|c|c|c|c|}
\hline \multirow[b]{2}{*}{ Tracks } & \multirow{2}{*}{${ }_{\text {distribution }}^{k_{z}}$} & \multirow{2}{*}{$\begin{array}{l}\text { Height of } \\
\text { ambiguity }\end{array}$} & \multicolumn{3}{|c|}{ Resolution } \\
\hline & & & Vertical $^{2}$ & $\begin{array}{c}\text { Slant } \\
\text { Range }^{3}\end{array}$ & Azimuth $^{3}$ \\
\hline 9 & $\begin{array}{l}\text { Uniform } \\
\text { distributed } \\
\text { from } 0 \text { to } \\
0.55\end{array}$ & $91 \mathrm{~m}$ & $11.4 \mathrm{~m}$ & $1.2 \mathrm{~m}$ & $0.6 \mathrm{~m}$ \\
\hline
\end{tabular}

${ }^{1} k_{z}$ is the vertical wavenumber for the center of the image, ${ }^{2}$ Rayleigh resolution, ${ }^{3}$ Before tomographic processing.

the DLR's F-SAR system [35] (see Fig. 4 bottom). The different parameters of the dataset are summarized in Table I. In 2016, airborne Lidar measurements were acquired over the same site (see Fig. 4 top).

As shown in Table I, the SAR single look complex (SLC) images have a resolution of $1.2 \mathrm{~m}$ in range and $0.6 \mathrm{~m}$ in azimuth. A multilook of $5 \times 11$ SLC samples in range and azimuth, respectively, is applied in the tomographic processing for the estimation of the covariance matrices, which leads to independent reflectivity profiles every $6 \times 6.6 \mathrm{~m}$. The Appendix summarizes the main tomographic processing steps employed.

Then, the significant peaks from each reflectivity profile are identified. In order to do so, several standard approaches have been tested. Since the local maxima we intend to identify in the reflectivity profiles have significantly higher amplitude than spurious peaks caused by noise (as it can be observed in Fig. 2), no meaningful differences in terms of performance between the methods have been found in this case. For the results reported in this paper, the profile is first convolved with a Gaussian wavelet at two scales. Within the wavelet coefficients, the ones with amplitude higher than the mean plus three times the standard deviation are accepted as actual peaks. As already mentioned, 


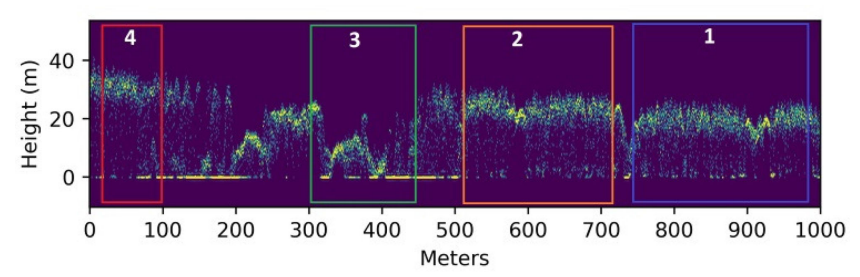

(a)

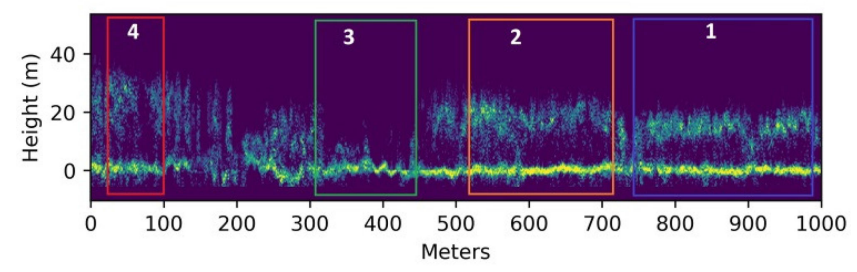

(b)

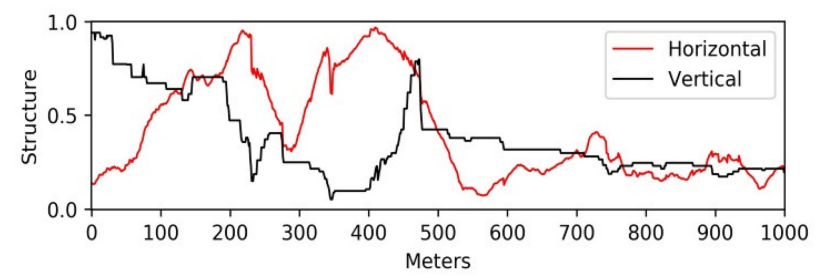

(c)

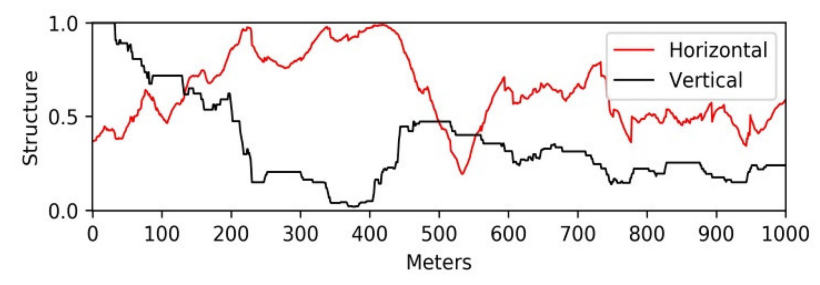

(d)

Fig. 5. Transect across the test site as observed through (a) the Lidar returns and (b) the peaks of the TomoSAR reflectivity profiles. Corresponding forest structure indices as estimated from (c) Lidar and (d) TomoSAR, following (3) and (4) (aggregated Lidar vertical profiles are treated as TomoSAR reflectivity profiles).

the identified peaks are projected on a geographical $1 \times 1 \mathrm{~m}$ grid before the estimation of the structure indices.

After choosing a scale, the forest structure indices are then computed at this scale by applying (3) and (4) from Section II. This is implemented by means of a sliding window, whose dimensions correspond to the chosen scale sliding through the samples in the geographical $1 \times 1 \mathrm{~m}$ grid. Note that, throughout the paper, the scale is set to $50 \times 50 \mathrm{~m}$ (except of Section III-D, where a multiscale analysis is performed).

A similar analysis has been carried out as well with Lidar data in the same test site. For the Lidar data, a return is recorded every $0.5 \times 0.5 \mathrm{~m}$. First, the 100 returns in $5 \times 5 \mathrm{~m}$ are combined to obtain an aggregated vertical profile. Then, each of these profiles is treated in the same way than the radar ones: The peaks are identified and the structures are estimated following (3) and (4) with a sliding window.

Fig. 5 shows a $1 \mathrm{~km}$ long transect along the site [indicated by the white line in Fig. 4 (top)], as seen by both the Lidar (a) and the TomoSAR (b) systems. At each position of the transect, the projection of the Lidar returns as well as the TomoSAR peaks across $20 \mathrm{~m}$ are represented. Higher values (yellowish colors) reflect the co-occurrence of numerous peaks at a given height, whereas lower values (bluish colors) imply that no peaks are detected there. It is worth clarifying here that Figs. 2 and 5, besides covering different sites, are showing different variables: Fig. 2 shows the normalized reflectivity, whereas Fig. 5 shows the aggregation of reflectivity peaks. Four areas are highlighted in this plot, numbered from 1 to 4, from East to West. For instance, Area 1 corresponds to a homogeneous monolayer stand of spruces. For this young stand, most of the peaks are concentrated in a limited range of heights and only a few ones appear beneath this dominant layer. In Area 2, the dispersion in the vertical distribution of peaks is slightly increasing, reflecting the transition to a multilayered stand. Area 3 is essentially constituted by very low vegetation, irregularly scattered in space. Finally, at the western part of the plot, Area 4 comprises an older stand, with a significantly higher structure complexity that is consistently reflected in a wider distribution of peaks. By comparing the distribution of the Lidar returns with one of the tomographic peaks along the transect, the differences between the different areas in this example appear clearer in the TomoSAR transect. The Lidar returns appear mostly concentrated at the top of the canopy, in a similar way all along the transect. For example, the main difference between Areas 4 and 1 is that the returns in Area 4 are a few meters higher, but they are not significantly more scattered in the vertical dimension than the ones in Area 1. This is reflected in the values of the horizontal and vertical forest structure indices in this transect [see Fig. 5(c) and (d)].

\section{B. Results}

The maps of horizontal and vertical structure are estimated in the site for the three different sources of data available at a structure scale of $50 \times 50 \mathrm{~m}$. First, the horizontal and vertical structures from the field data are computed following (1) and (2), respectively. Then, the horizontal and vertical structures for the two sources of remote sensing data are estimated according to (3) and (4). The resulting maps of horizontal and vertical forest structure are shown in Figs. 6 and 7. Despite several local dissimilarities, the general spatial patterns for the three maps are similar. In terms of horizontal structure, the test site is quite homogeneous, apart from the central area with low and scattered vegetation. In terms of vertical structure, a gradient of increasing complexity from East to West is distinctly noticeable.

The discrepancies between the different maps are mainly due to differences in resolution and viewing geometries affecting in particular stand borders. Such an example is shown in Fig. 8, which shows the area within the square indicated in Fig. 4. When the vertical structure is estimated from field data, a local maximum can be observed in the map. When estimated from Lidar, this maximum is spread over a much larger area and when estimated from TomoSAR, no vertical structure maxima are retrieved in this point. According to field data, in this area, as shown in Fig. 8, there are three isolated tall trees surrounded by very low vegetation. This should increase locally vertical structure, but the impact of these trees in the three maps appears to be very different. In the field data, in most of the sliding 

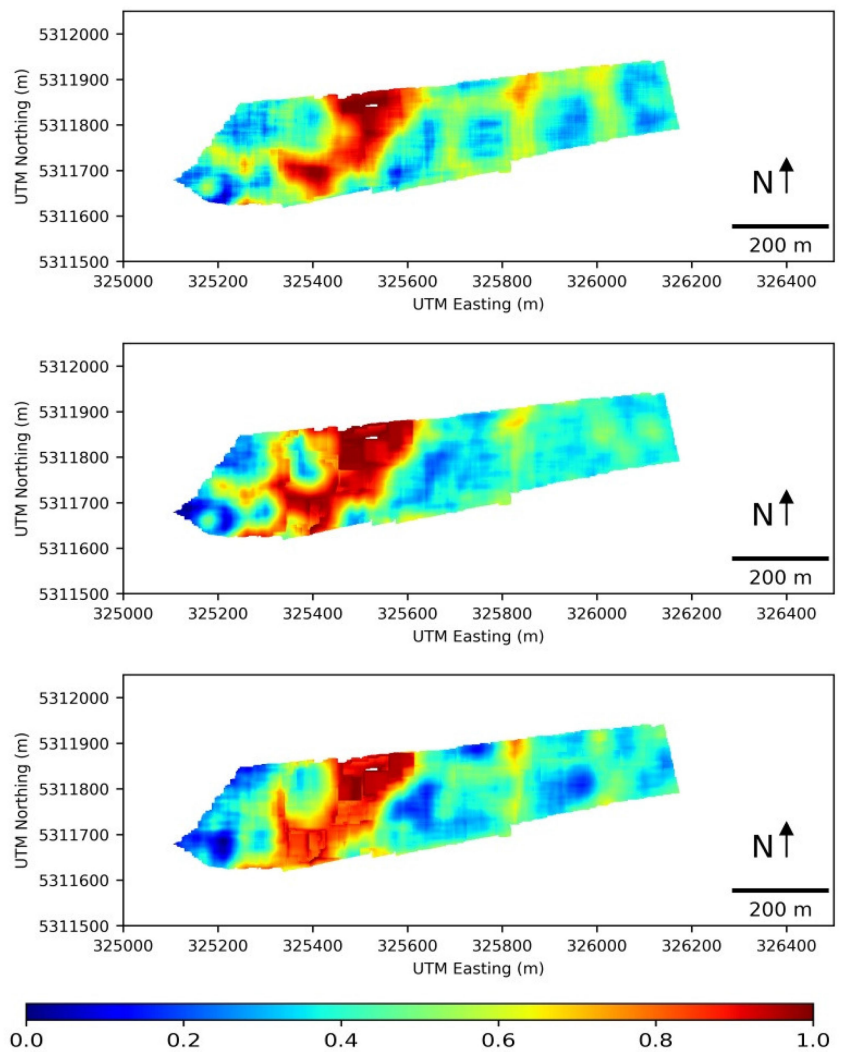

Fig. 6. Maps of horizontal forest structure, as estimated from (top) field, (middle) Lidar and (bottom) TomoSAR data.

windows covering these trees, the number of tall trees is low with respect to the number of smaller trees. On the contrary, for the Lidar data, since big trees produce more returns than the smaller ones, their presence will be exaggerated and spread over the area of the structure window. Lastly, in the tomographic SAR reconstruction, the tall trees could not be detected (see Fig. 8), essentially because of insufficient spatial resolution after multilook $(6 \times 6.6 \mathrm{~m})$.

The correlations between the different maps are shown in Fig. 9. Correlations are slightly better for the horizontal than for the vertical structure estimators, which are reasonable because the vertical measure involves a second-order moment, whereas the horizontal one reflects first-order statistics. It can be observed from the maps and the correlation plots that across the site, structure types are unevenly distributed: Both for the horizontal and the vertical, most of the site exhibits similarly low values and very high values are concentrated in a small area. Also, especially for the vertical structure, transitions between areas with different structure are smoother than with Lidar and TomoSAR. In this sense, the higher sensitivity of the field data to vertical structure variations is due to the difference in accuracy of the measurements involved: Vertical structure estimated from field data according to (2) is sensitive to variations of dbh in the range of $\mathrm{cms}$, whereas Lidar and especially TomoSAR are constrained by the vertical resolution of the system, which is on the order of several meters. Besides this, it can be observed that
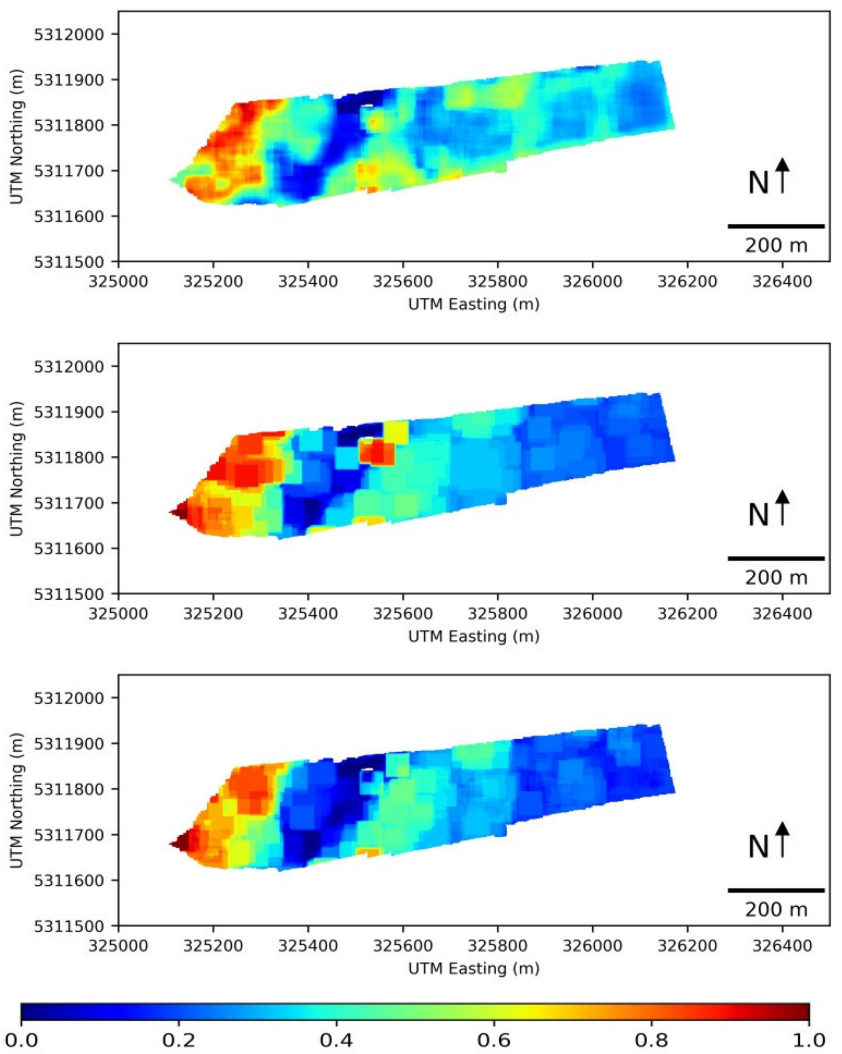

Fig. 7. Maps of vertical forest structure, as estimated from (top) field, (middle) Lidar, and (bottom) TomoSAR data.

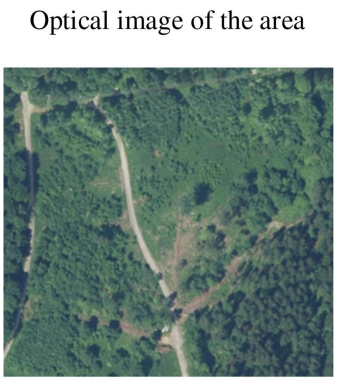

Map of Lidar heights

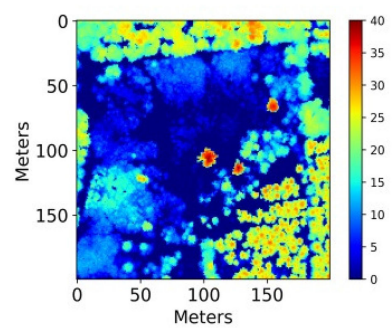

Position of the trees sampled with its $\mathrm{dbh}$

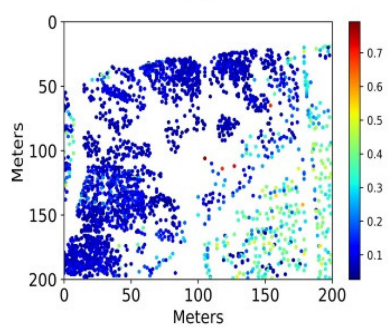

Height of the highest peak in the TomoSAR reflectivities

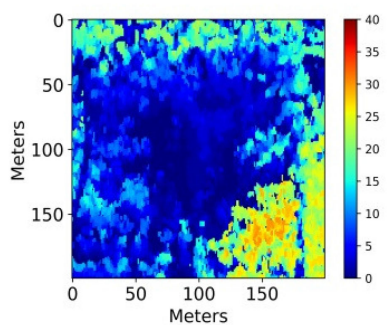

Fig. 8. Area in the white square in Fig. 4.

the structure estimated from Lidar shows a better correlation with the field data than the one estimated from TomoSAR for the horizontal dimension $(r=0.87$ versus $r=0.83)$, but slightly worse for the vertical one ( $r=0.75$ versus $r=0.77)$. 

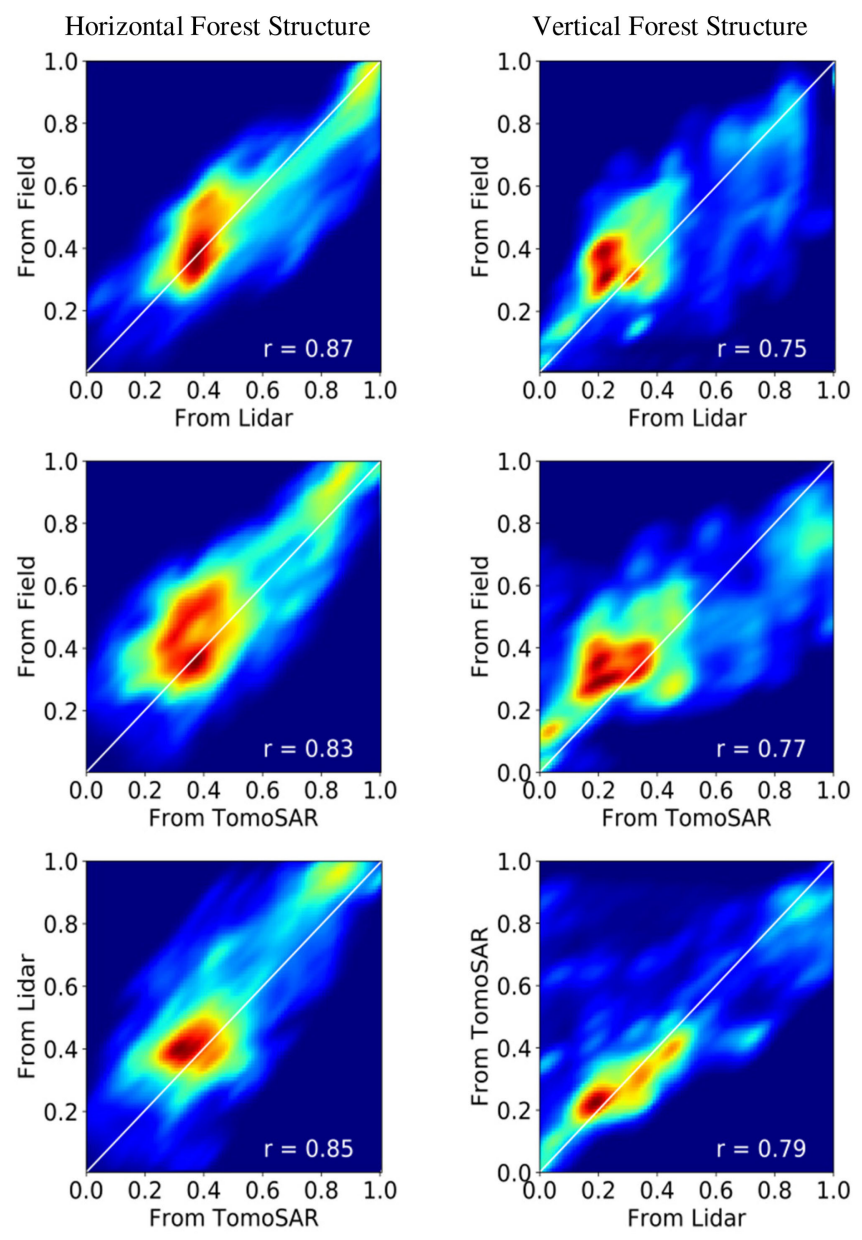

Fig. 9. Correlations between horizontal (left column) and vertical (right column) forest structures as estimated from Field, Lidar, and TomoSAR data.

\section{3-D Versus Height Only Forest Structure Estimation}

In order to assess the advantages of employing tomographic SAR for forest structure estimation with respect to only height in the same conditions of spatial resolution, a simple test is carried out by estimating forest structure only taking into account the highest peak for each range and azimuth position instead of the complete vertical distribution of peaks. It is assumed here that the highest reflectivity peak is the closest one to the top of the canopy. Using the highest peaks only, horizontal and vertical structures can be estimated according to (3) and (4) following the same procedure as before. The maps of horizontal and vertical structure obtained are shown in Fig. 10. A lower sensitivity when compared to the structures, estimated from the whole set of peaks (see Figs. 6 and 7), can be observed essentially in the high vertical structure region, in the West part of the site. This is reflected as well in the correlation plots shown in Fig. 11: Vertical structure is underestimated when considering only the highest peak and the underestimation is more important for higher structure values.

Four areas considered uniform in terms of structure according to field data and optical imagery available are defined. These four areas are shown in Fig. 4 (top). From East to West, the area in the blue polygon is a monolayer dense stand. The orange
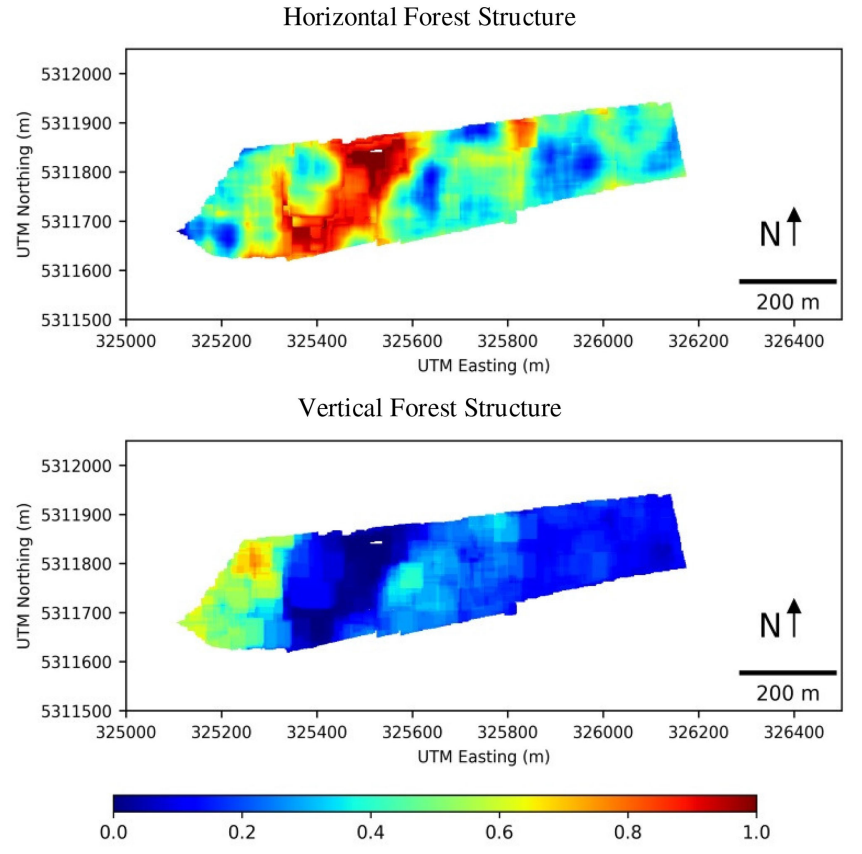

Fig. 10. Maps of forest structure estimated from the highest peak of the reflectivity profiles. (Top) Horizontal and (bottom) vertical forest structure.
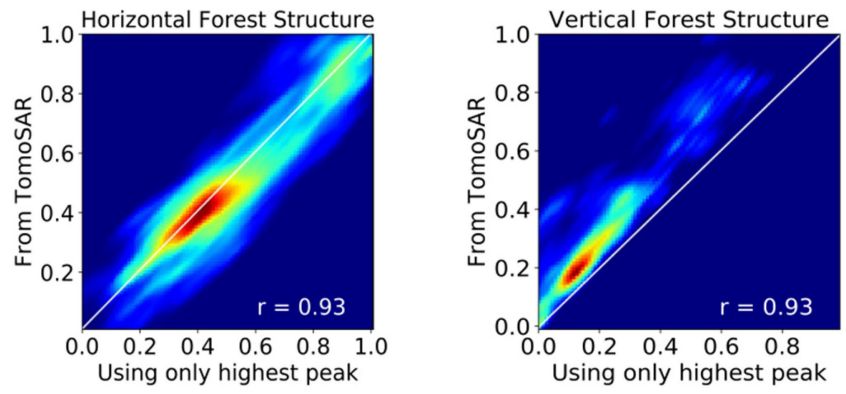

Fig. 11. Correlations between the structures as estimated from the complete set of peaks of the TomoSAR reflectivity profiles and from only the highest one.

polygon is of similar structure besides a few older trees to its western border increasing complexity. The green polygon is a very heterogeneous area with only a few scattered trees. Lastly, the red area comprises an older stand with a larger diversity in terms of trees dimensions and thus higher vertical structure.

Fig. 12 shows the distribution of the indices in the HV plane for these four areas, considering the whole set of peaks and considering only the highest peak, at $50 \times 50 \mathrm{~m}$. When only the highest peak is considered, the reduction in sensitivity in the vertical dimension induces a vertical shrinkage of the signatures of the different areas, supporting the reduction of sensitivity to vertical structure observed from the maps in Fig. 10 and the correlation plots in Fig. 11.

\section{Discussion on Scale}

As already mentioned in Section II-B2, forest structure is inherently linked to scale and as a consequence the estimation of forest structure might vary with scale. Also, the measure 
HV plane of forest structure indices estimated from the whole set of peaks of the TomoSAR reflectivities

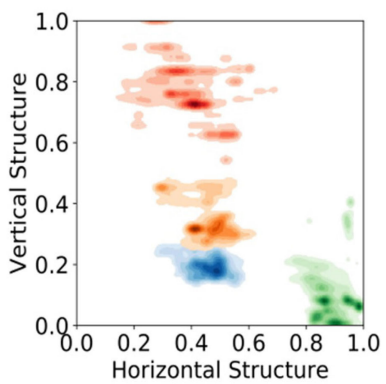

HV plane of forest structure indices estimated from the highest peak only

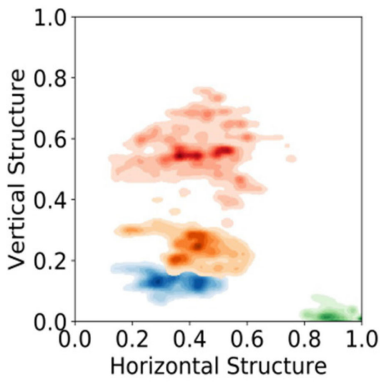

Fig. 12. Signatures of the horizontal and vertical forest structure indices in the HV plane for four different polygons at different growth stages (young monolayer stand in blue, older mainly monolayer stand in orange, heterogeneous area with scattered trees in green, and multilayered stand in red), estimated using the whole set of peaks of the TomoSAR reflectivities (left) and using only the highest one (right), at $50 \times 50 \mathrm{~m}$.

of forest structure can be considered meaningful only within a range of scales. This range is bounded on the lower limit by the spatial variability of the scene and the spatial resolution of the sampling source. In fact, since the indices proposed rely on statistical measures, in order to ensure statistical significance, a minimum number of samples is required, both in terms of trees within the area considered and in terms of independent SAR observations. Too fine scales are not adequate to provide statistically significant values and, even within a uniform area, estimated forest structure may differ from sample to sample. On the other extreme, the upper limit of the valid scales is determined by the maximum correlation length of the stand. If the scales considered are larger than the size of the areas regarded as being uniform, the resulting estimates result from the mixing of diverse structures types and are therefore biased. Furthermore, besides the statistical considerations, ecological significance should also be accounted for. In that sense, it should be noted that the relations between forest species and 3-D vegetation structure may occur at spatial scales finer as 1 ha 5 and therefore scales of 1 ha or finer are required for many ecological applications.

In order to illustrate the evolution of structure estimates across scales in the site considered, the indices of horizontal and vertical forest structure for the three sources available (field measurements, Lidar, and TomoSAR data) are estimated according to (1)-(4) at scales ranging from $25 \times 25 \mathrm{~m}$ to $150 \times 150 \mathrm{~m}$. The distribution of the values of estimated forest structure in the $\mathrm{HV}$ plane for three different scales $(25 \times 25 \mathrm{~m}, 75 \times 75 \mathrm{~m}$, and $150 \times 150 \mathrm{~m}$ ) in the four areas regarded as uniform in terms of structure and defined in the previous section is shown in Fig. 13.

Regardless of the source of data, it can be observed that at a scale of $25 \times 25 \mathrm{~m}$, the dispersion of values within polygons considered uniform in terms of structure is high. It can thus be deduced that especially in the red polygon with the highest vertical structure, cells of $25 \times 25 \mathrm{~m}$ are too fine to sample its representative diversity. This large dispersion would hamper as well the classification of polygons, since the distribution of their values of horizontal and vertical structures appears close to each other or even overlapped in the HV plane. When the scale

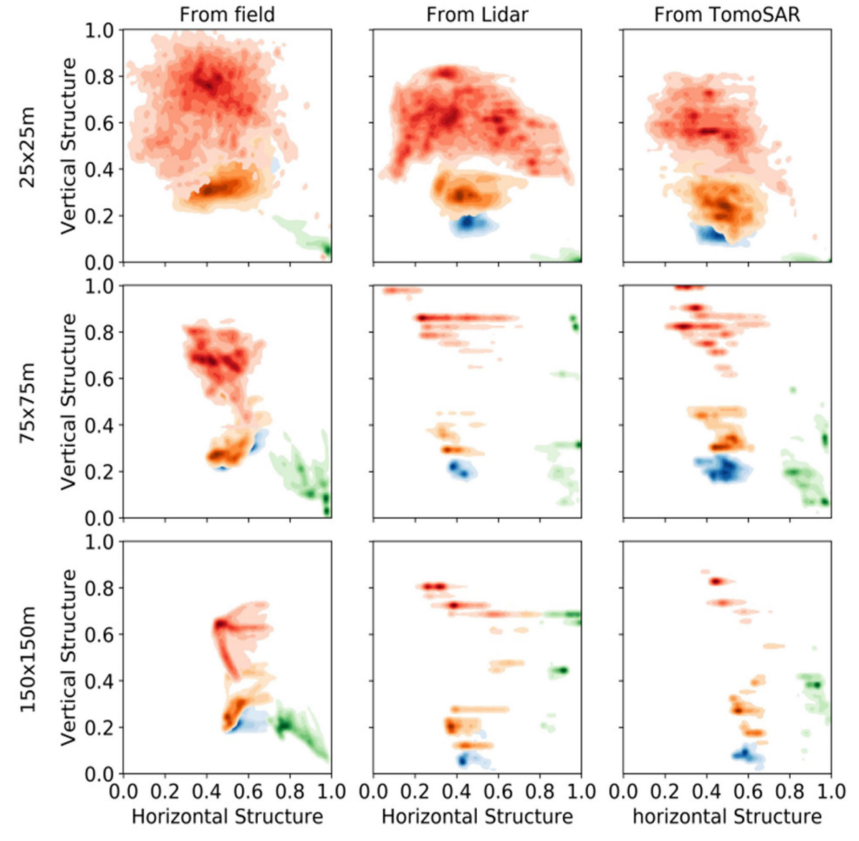

Fig. 13. Signatures of the horizontal and vertical forest structure indices in the HV plane for four different polygons at different growth stages (young monolayer stand in blue, older mainly monolayer stand in orange, heterogeneous area with scattered trees in green and multilayered stand in red), as estimated from field, Lidar, and TomoSAR data, at three different scales.

increases to $75 \times 75 \mathrm{~m}$, the dispersion of values within polygons is significantly reduced and the distributions for the different polygons drift apart from each other, favoring classification based on structure characterization. The standard deviation within the predefined polygons further decreases if the scale increases to $150 \times 150 \mathrm{~m}$, for the three sources of data. However, the four different signatures start to shrink again and become meaningfully less distinct. In fact, the elongated morphology of the test site considered in this paper together with its rapid spatial variability (see Fig. 4) allows only a few windows larger than 1 ha still covering uniform trees distributions.

\section{CONCLUSION}

A framework for qualitative and quantitative forest structure estimation from tomographic SAR measurements at L-band has been proposed. It is based on a pair of complementary indices for the characterization of horizontal and vertical structure. In order to ensure the physical interpretation and ecological relevance, the indices have been derived in correspondence to structure indices already established in forestry and ecology studies. The fact that the indices used in forestry and ecology are defined in terms of individual tree parameters while conventional SAR measurements are not able - in terms of spatial resolution- to distinguish single trees had to be accounted. A methodology to derive the two indices from 3-D radar reflectivity reconstructed by tomographic SAR techniques has been proposed and described step by step. The methodology relies on the evaluation of the location of the (local) maxima of the reconstructed vertical reflectivity profiles. The main reason for this is that the locations of the reflectivity maxima are less prone to variations induced 
by nonstructural effects as for example by changing seasonal and weather conditions than the reflectivity values itself.

The proposed methodology has been applied on airborne tomographic SAR data acquired at L-band over the temperate forest of Traunstein in Germany and validated against the structure indices obtained from airborne Lidar and field inventory data. The validation of the forest structure maps obtained from the radar data against the ones derived from the field inventory data revealed a meaningful correspondence. Reaching a good agreement between the maps generated from the two data sources is necessary to ensure the incorporation of the estimations generated by SAR systems in ecology studies, for example, through assimilation into existing models.

The framework as defined by the two indices allows:

1) the systematic interpretation of the 3-D radar reflectivity in terms of physical forest structure. This is not always an intuitive task because of the dependency of radar reflectivity on system parameters as well as on the geometric and dielectric properties of the forest;

2) the direct comparison of forest structure estimates from field inventory data to estimates derived from radar and even Lidar measurements.

Indeed, the proposed framework has proven to be suitable for Lidar measurements. The forest structure maps as estimated by means of radar and Lidar are comparable, despite the differences in resolution and acquisition geometry. This not only supports the significance of the proposed framework but also establishes a basis to combine the information provided by radar and Lidar.

It should be noted here that the quantitative characterization of forest structure by means of the proposed framework unavoidably incurs a loss of structure information, since a 3-D notion is projected into a 2-D space defined by the two indices, whose orthogonality is not guaranteed in any case (or scale). In this sense, further validation is necessary to entirely assess the limitations of the framework proposed when it comes to a complete representation of forest structure.

In order to assess the value added by the availability of full 3-D information when compared to the information provided by forest height alone, forest structure maps generated by accounting only the highest maximum of the reflectivity profiles have been generated. The considerable loss of sensitivity to vertical structure variations in these maps reinforces the importance of 3-D radar reflectivity estimates.

Finally, the evolution of the obtained forest structure estimates has been evaluated over a range of spatial scales. The main result was the understanding that the choice of scale has to be meaningful with respect to the structural complexity of the underlying stand or forest. For a complex highly heterogeneous stand, estimates at very fine scales (smaller than $25 \times 25 \mathrm{~m}$ ) are affected by a high dispersion. The dispersion decreases rapidly toward $50 \times 50 \mathrm{~m}$ and beyond which is in agreement with the common understanding that the spatial distribution of trees at 0.5 to 1 ha scales renders a meaningful estimate of the complexity of a stand. The upper bound is given by the maximum scale at which the stand can still be considered uniform in terms of structure.
Further validation and possible refinements of the proposed methodology require sites and data with spatial and temporal diversity, i.e., a variety of forest scenarios with different characteristics and temporal variability. Regarding spatial variability, the proposed methodology has been already successfully validated in a number of tropical test sites in Gabon [36]. Temporal variability is important for assessing the sensitivity to temporal and spatial changes in forest structure and the robustness to nonstructural variations. In this context the proposed methodology has been tested on a time series of tomographic acquisitions conducted over temperate forests and proven to be robust enough to nonstructural variations and sensitive enough for tracking forest structure changes due to management practices [34].

\section{APPENDIX \\ PRINCIPLES OF SAR TOMOGRAPHY AND COMPRESSIVE SENSING}

Consider a tomographic acquisition composed by $M$ (repeatpass) tracks over the same scene. Given a range and azimuth coordinate, the SLC pixel amplitudes $y_{m}, m=1, \ldots, M$ of a single-polarimetric channel can be arranged in a column vector $y$ as follows:

$$
y=\left[y_{1}, y_{2}, \ldots, y_{M}\right]^{T} .
$$

In the multipolarimetric case, the same operation can be repeated for each one of the $P$ available polarimetric channels ( $P=3$ for a fully polarimetric acquisition). Then, a $P M$ dimensional SLC data vector $y_{p}$ is obtained by stacking the single-polarimetric data vectors one on top of the other.

Since each image is acquired from a slightly different angular position, a scatterer at a given height $z$ introduces a phase difference among the acquisitions. The vertical sensitivity of the phase difference between two acquisitions is expressed by means of the vertical wavenumber defined as follows [37]:

$$
k_{z}=\frac{4 \pi B_{\perp}}{\lambda r \sin (\theta)}
$$

where $\lambda$ is the wavelength, $r$ is the distance to the scatterer, $\theta$ is the incidence angle, and $B_{\perp}$ is the orthogonal distance between the tracks corresponding to the two acquisitions projected in the direction orthogonal to the line of sight. Let $k_{z \max }$ be the wavenumber with maximum $B_{\perp}$, and $k_{z \text { min }}$ the one with minimum $B_{\perp}$. The value of $k_{z \max }$ is related to the Rayleigh vertical resolution $\delta_{z}$ as [37]

$$
\delta_{z}=\frac{2 \pi}{k_{z \max }}
$$

which is conventionally used as a reference in SAR tomography. $k_{z \text { min }}$ gives the nonambiguous height interval $h_{\mathrm{amb}}$ as [37]

$$
h_{\mathrm{amb}}=\frac{2 \pi}{k_{z \min }} .
$$

In order to avoid ambiguities in the estimated vertical distribution of the backscattered power (reflectivity profile), $h_{\mathrm{amb}}$ should be higher than the maximum forest height of the scene. 
The phase differences of each of the acquisitions are collected in the so-called z-dependent steering vector at a given height $z$ defined as follows:

$$
a(z)=\left[1, e^{j k_{z_{2}} z}, e^{j k_{z_{3}} z}, \ldots, e^{j k_{z_{M}} z}\right]^{T}
$$

where $k_{z_{m}}, m=1, \ldots, M$, is the vertical wavenumber for the $m$ th acquisition with respect to the reference one, here placed in correspondence of the first image for which it results $k_{z 1}=0$. In the multipolarimetric case, the $(P M \times P M)$-dimensional covariance matrix $R$ associated to $y_{p}$ is as follows:

$$
R=E\left\{y_{p} y_{p}{ }^{H}\right\} \text {. }
$$

In (A.6), $E\{\cdot\}$ denotes the statistical expectation, and $(\cdot)^{H}$ the Hermitian (i.e., transpose conjugate) operator. It should be noted that the estimation of the expectation of a stochastic process requires several realizations of that process, which is generally not available in real datasets. Therefore, under the assumption of ergodicity, the statistical expectation in (A.6) is approximated by averaging neighboring pixels within a range-azimuth (multilook) cell.

Several (model-based and not) tomographic algorithms can be employed to estimate the reflectivity profiles using $R$ and $a(z)$ [31], [38] on a set of heights $\left\{z_{i}\right\}_{i=1}^{H}$ within an interval of interest. In the general multipolarimetric case, it is of interest to estimate a set of $(P \times P)$-dimensional polarimetric covariance matrices $\left\{T\left(z_{i}\right)\right\}_{i=1}^{H}$. Their $P$ diagonal elements collected for the $H$ heights form the reflectivity profiles in the $P$ polarimetric channels. Different algorithms are characterized by different height resolution capabilities beyond the Rayleigh limit (A.3). However, the improvement in the achievable resolution can intrinsically reduce the radiometric accuracy, especially in the imaging of volume scatterers.

The estimation of the reflectivity profiles is based on the inversion of the Fourier relationship existing between them and the covariances in (A.6) [11], [37]. Following the derivation in [39], such relationship can be expressed by means of a linear system as follows:

$$
R^{\prime}=\phi T^{\prime}
$$

In (A.7) $R^{\prime}$ is $\left(M^{2} \times P^{2}\right)$-dimensional matrix resulting from a proper permutation of the elements of the polarimetricmultibaseline covariance matrix (A.6): Each row represents for each of the $M^{2}$ interferometric pairs, the different $P^{2}$ polarization combinations, after ordering the elements of the $(P \times P)$-dimensional polarimetric matrices as vectors. Further details on the permutation are given in [39]. $\phi$ is an $\left(M^{2} \times H\right)$ dimensional matrix obtained by collecting the steering vectors (A.5) calculated for the $H$ heights [39]. Finally, $T^{\prime}$ is an $\left(H \times P^{2}\right)$-dimensional matrix that contains the elements of the unknown matrices $\left\{T\left(z_{i}\right)\right\}_{i=1}^{H}$ on each row [39]. Since in an usual tomographic configuration the number of acquisitions is limited, it results in $M^{2} \ll H$. As a consequence, the linear system in (A.7) is highly underdetermined.

In order to estimate the vertical reflectivity profiles by solving the linear system in (A.7), in this paper we make use of an algorithm based on CS [40]. Essentially, the CS theory states that a signal can be reconstructed under the Nyquist sampling rate provided that it is sparse, meaning that it has a low number of nonzero coefficients, and the matrix $\phi$ fulfills the restricted isometry property [40]. Thus, a CS algorithm can cope with the underdetermination of the system (A.7) and can resolve scatterers at heights closer than the resolution limit in (A.3).

In general, the sparsity condition is too restrictive and most of the signals of interest do not fulfill it. However, the application of CS can be easily extended to compressible signals, which are signals that produce a sparse representation when projected in a fixed sparsifying basis.

Since some level of correlation is expected between adjacent rows in $T^{\prime}$ (i.e., consecutive heights), it is reasonable to assume that a sparse representation of $T^{\prime}$ may be obtained through a projection on a given basis. This has already been explored in [41], where it has been proven that the projection into an appropriate wavelet basis provides a sparse expansion of the reflectivity profiles in a forest scenario. Hence, by denoting with $w$ the $(H \times H)$-dimensional matrix of the wavelet projection, the vector of the sparse wavelet coefficients $\alpha$ results in the following:

$$
\alpha=w T^{\prime}
$$

With this, (A.7) can be rewritten as follows:

$$
R^{\prime}=\phi w^{-1} \alpha \text {. }
$$

In this paper, we suggest to estimate $\alpha$ by solving (A.9) through a disciplined convex programming, which can be formalized by means of the following constrained minimization problem:

$$
\min \|\tilde{\alpha}\|_{2,1} \text { subject to }\left\|R^{\prime}-\phi w^{-1} \tilde{\alpha}\right\|_{F} \leq \varepsilon
$$

under the additional condition that every row of $T^{\prime}$ (i.e., each polarimetric covariance matrix) must be positive semidefinite. In (A.10), $\tilde{\alpha}$ are the estimated wavelet coefficients, $\varepsilon$ is an upper bound for the error allowed in the estimation, $\|\cdot\|_{2,1}$ and $\|\cdot\|_{F}$ are a mixed $(2,1)$ and a Frobenius norm, respectively. In the scope of this paper, the proposed approach has been implemented in Python and employs the CVXPY packages [42]. With the estimated coefficients $\tilde{\alpha}$ from (A.10), the estimated polarimetric covariance matrices $\tilde{T}^{\prime}$ and finally the reflectivity profiles can be obtained.

\section{ACKNOWLEDGMENT}

The authors would like to thank the Municipal Forest Administration of the City of Traunstein for making this project possible on their estate property and the F-SAR team for its invaluable effort during the data acquisition and processing. The Traunstein Forest Dynamics Plot is a collaborative project of the Chair for Forest Growth and Yield, Technische Universität München, the Department of Ecological Modelling at the Helmholtz Centre for Environmental Research UFZ, Leipzig, and the Microwaves and Radar Institute at the German Aerospace Center - DLR, Oberpfaffenhofen.

\section{REFERENCES}

[1] M. Snyder, "What is forest stand structure and how is it measured?" Northern Woodlands, Lyme, NH, USA, Issue no. 64, p. 15, 2010. 
[2] T. A. Spies, "Forest structure: A key to the ecosystem," Northwest Sci., vol. 72, pp. 34-36, 1998.

[3] F. G. Halll et al., "Characterizing 3D vegetation structure from space: Mission requirements," Remote Sens. Environ., vol. 115, pp. 2753-2775, 2011.

[4] N. Brokaw and R. Lent, "Vertical Structure," in Maintaining Biodiversity in Forest Ecosystems. Cambridge, U.K.: Cambridge Univ. Press, 1999, pp. 373-399.

[5] C. McElhinny, P. Gibbons, C. Brack, and J. Bauhus, "Forest and woodland stand structural complexity: Its definition and measurement," Forest Ecol. Manage., vol. 218, pp. 1-24, 2005.

[6] F. J. Bohn and A. Huth, "The importance of forest structure to biodiversityproductivity relationships," Roy. Soc. Open Sci., vol. 4, 2017, Art. no. 160521 .

[7] K. M. Bergen et al., "Remote sensing of vegetation 3-D structure for biodiversity and habitat: Review and implications for lidar and radar spaceborne missions," J. Geophys. Res., vol. 114, 2009, Art. no. G003E06.

[8] S. Goetz, D. Steinberg, R. Dubayah, and B. Blair, "Laser remote sensing of canopy habitat heterogeneity as a predictor of bird species richness in an eastern temperate forest," Remote Sens. Environ., vol. 108, pp. 254-263, 2007.

[9] W. Turner, S. Spector, N. Gardiner, M. Fladeland, E. Sterling, and M. Steininger, "Remote sensing for biodiversity science and conservation," Trends Ecol. Evol., vol. 18, pp. 306-314, 2003.

[10] S. Frolking, M. W. Palace, D. B. Clark, J. Q. Chambers, H. H. Shugart, and G. C. Hurtt, "Forest disturbance and recovery: A general review in the context of spaceborne remote sensing of impacts on aboveground biomass and canopy structure," J. Geophys. Res., vol. 114, 2009, Art. no. G00E02.

[11] S. Cloude and K. Papathanassiou, "Three-stage inversion process for polarimetric SAR interferometry," IEE Proc., Radar Sonar Navig., vol. 150, no. 3, pp. 125-134, 2003.

[12] D. Ho Tong Minh et al., "TropiScat: Multi-temporal multi-polarimetric tomographic imaging of tropical forest," in Proc. Int. Geosci. Remote Sens. Symp., Munich, Germany, Jul. 2012, pp. 7051-7054.

[13] M. Pardini, K. Papathanassiou, and F. Lombardini, "Impact of dielectric changes on L-band 3-D reflectivity profiles of forest volumes," IEEE Trans. Geosci. Remote Sens., to be published, doi: 10.1109/TGRS.2018.2850357.

[14] T. Le Toan et al., "The biomass mission: Mapping global forest biomass to better understand the terrestrial carbon cycle," Remote Sens. Environ., vol. 115, no. 11, pp. 2850-2860, 2011.

[15] A. Moreira et al., "Tandem-L: A highly innovative bistatic SAR mission for global observation of dynamic processes on the earth's surface," IEEE Geosci. Remote Sens. Mag., vol. 3, no. 2, pp. 8-23, Jun. 2015.

[16] P. Clark and C. Evans, "Distance to nearest neighbor as a measure of spatial relationships in populations," Ecology, vol. 35, no. 4, pp. 445-452, 1954.

[17] F. Pastorella and A. Paletto, "Stand structure indices as tools to support forest management: An application in Trentino forests (Italy)," J. Forest Sci., vol. 59, no. 4, pp. 159-168, 2013.

[18] E. K. Zenner and D. Hibbs, "A new method for modeling the heterogeneity of forest structure," Forest Ecology Manage., vol. 129, pp. 75-87, 2000.

[19] A. Pommerening, "Approaches to quantifying forest structures," Forestry, vol. 75, pp. 305-324, 2002.

[20] H. Pretzsch, Forest Dynamics, Growth and Yield. Berlin, Germany: Springer-Verlag, 2009.

[21] M. del Rio et al., "Characterization of structure, dynamics, and productivity of mixed-species stands: review and perspectives," Eur. J. Forest Res., vol. 135, no. 1, pp 23-49, 2016

[22] C. D. Oliver and B. C. Larson, Forest Stand Dynamics. New York, NY, USA: Wiley, 1996.

[23] L. Reineke, "Perfecting a stand-density index for evenaged forests," $J$. Agric. Res., vol. 46, no. 7, pp. 627-638, 1933.

[24] R. O. Curtis and D. D. Marshall, "Why quadratic mean diameter?" Western J. Appl. Forestry, vol. 15, pp. 137-139, 2000.

[25] H. Pretzsch and P. Biber, "A ee-evaluation of Reineke's rule and stand density index," Forest Sci., vol. 51, no. 4, pp. 304-320, 2005.

[26] J. Liang, "Effects of diversity of tree species and size on forest basal growth, recruitment, and mortality," Forest Ecol. Manage., vol. 243, no. 1, pp. 116-127, 2007.

[27] I. Barbeito, F. Montes, and I. Canellas, "Evaluating the behavior of vertical structure indices in Scots pine forests," Ann. Forest Sci., vol. 66, no. 7, pp. 1-10, 2009.
[28] M. Brolly and I. H. Woodhouse, "Vertical backscatter profile of forests predicted by a macroecological plant model," Int. J. Remote Sens., vol. 34, no. 4, pp. 1026-1040, 2013.

[29] L. Thirion, E. Colin, and C. Dahon, "Capabilities of a forest coherent scattering model applied to radiometry, interferometry, and polarimetry at P- and L-band," IEEE Trans. Geosci. Remote Sens., vol. 44, no. 4, pp. 849-862, Apr. 2006.

[30] Y. Lin and K. Sarabandi, "A Monte Carlo coherent scattering model for forest canopies using fractal-generated trees," IEEE Trans. Geosci. Remote Sens., vol. 37, no. 1, pp. 440-451, Jan. 1999.

[31] O. Frey and E. Meier, "Analyzing tomographic SAR data of a forest with respect to frequency, polarization, and focusing technique," IEEE Trans. Geosci. Remote Sens., vol. 49, no. 10, pp. 3648-3659, Oct. 2011.

[32] V. Cazcarra-Bes, M. Tello, M. Pardini, and K. Papathanassiou, "Assessment of tomographic SAR processing techniques for forest structure estimation," in Proc. Eur. Conf. Synthetic Aperture Radar, Aachen, Germany, Jun. 2018, pp. 708-713.

[33] E. Assmann, The Principles of Forest Yield Study. Studies in the Organic Production, Structure, Increment and Yield of Forest Stands. Oxford, U.K. Pergamon Press, 1970.

[34] V. Cazcarra-Bes, M. Tello-Alonso, R. Fischer, M. Heym, and K. Papathanassiou, "Monitoring of forest structure dynamics by means of $\mathrm{L}$ band SAR tomography," Remote Sens., vol. 9, no. 12, 2017, Art. no. 1229.

[35] R. Horn et al., "SAR - DLR's new multifrequency polarimetric airborne SAR," Proc. IEEE Int. Geosci. Remote Sens. Symp., Cape Town, South Africa, Jul. 2009, pp. II-902-II-905.

[36] M. Pardini, M. Tello, V. Cazcarra-Bes, K. Papathanassiou, and I. Hajnsek, "L- and P-band 3-D SAR reflectivity profiles vs. lidar waveforms: The AfriSAR case," IEEE J. Sel. Topics Appl. Earth Obs. Remote Sens., to be published, doi: 10.1109/JSTARS.2018.2847033.

[37] A. Reigber and A. Moreira, "First demonstration of airborne SAR tomography using multibaseline L-band data," IEEE Trans. Geosci. Remote Sens., vol. 38, no. 5, pp. 2142-4152, Sep. 2000.

[38] S. Tebaldini, "Single and multipolarimetric SAR tomography of forested areas: A parametric approach," IEEE Trans. Geosci. Remote Sens., vol. 48, no. 5, pp. 2375-2387, May 2010

[39] E. Aguilera, M. Nannini, and A. Reigber, "A data-adaptative compressed sensing approach to polarimetric SAR tomography of forested areas," IEEE Trans. Geosci. Remote Sens. Lett., vol. 10, no. 3, pp. 543-547, May 2013

[40] E. Candès, J. Romberg, and T. Tao, "Robust uncertainty principles: Exact signal reconstruction from highly incomplete frequency information," IEEE Trans. Inf. Theory, vol. 52, no. 2 pp. 489-509, Feb. 2006.

[41] E. Aguilera, M. Nannini, and A. Reigber, "Wavelet-based compressed sensing for SAR tomography of forested areas," IEEE Trans. Geosci. Remote Sens., vol. 51, no. 12, pp. 5283-5295, Dec. 2013.

[42] S. Diamond and S. Boyd, "CVXPY: A python-embedded modeling language for convex optimization," J. Mach. Learn. Res., vol. 17, no. 83, pp. 1-5, 2016.

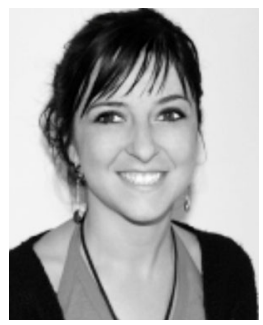

Marivi Tello (M'04) received the M.Sc. degree in electrical engineering and the Ph.D. degree in signal theory and communications from the Technical University of Catalonia, Barcelona, Spain, in 2003 and 2011, respectively.

Between 2010 and 2012, she was with the Catalan Institute of Climate Sciences (IC3). Between 2012 and 2013, she was awarded a Beatriu de Pinos fellowship and carried out a Postdoc at the Royal Netherlands Meteorological Institute (KNMI) in Utrecht, The Netherlands. In 2013, she joined the Microwaves and Radar Institute (HR) of the German Aerospace Center (DLR). She received a Marie Curie fellowship in 2014.

Her research interests include the field of signal processing, applied mainly to synthetic aperture radar data, but also to other types of signals such as climate variables. Currently, her main research activities are focused on methods for SAR tomography inversion and in the estimation of forest structure from TomoSAR data.

Dr. Tello was the recipient of the third prize to the best student paper at IGARSS in 2008, with a paper introducing a novel method of SAR focusing based on Compressive Sensing, and the Extraordinary Doctoral Thesis Award, by the Technical University of Catalonia (UPC) in 2013. 


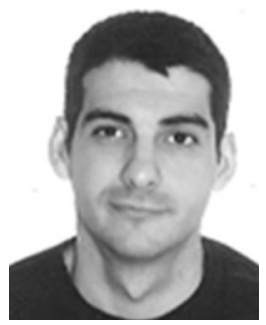

Victor Cazcarra-Bes was born in Barcelona, Spain in 1985. He received the B.S. degree in sound and image engineering from the University of Alicante, Alicante, Spain, in 2007, and the M.S. degree in telecommunication engineering from the University Miguel Hernández, Elche, Spain, in 2010.

Since 2014, he is with the Department of the Microwaves and Radar Institute, German Aerospace Center (DLR), Wessling, Germany, as a member of the Information retrieval research group. His main general research interests include the area of synthetic aperture radar signal processing for forest applications. He focuses on the processing algorithms of multibaseline polarimetric synthetic aperture radar data and the development of new algorithms to obtain 3-D forest structure.

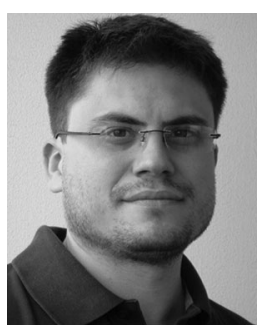

Matteo Pardini (S'02-M'10) received the M.Eng. degree (cum laude) in telecommunication engineering and the Ph.D. degree in information engineering from the University of Pisa, Pisa, Italy, in 2006 and 2010 , respectively.

In January 2010, he joined the Radar Concepts department, Microwaves and Radar Institute, German Aerospace Center (DLR), Wessling, Germany, as a Research Scientist, after a visiting research period from August to December 2009. In 2017, he was a Visiting Scientist with the Department of Geographical Sciences, University of Maryland, College Park, MD, USA. His main research interests include synthetic aperture radar (SAR) tomographic and polarimetric interferometric processing for 3-D bio/geophysical information extraction over natural volumes (forest, agriculture, ice), as well as in SAR mission design and SAR mission performance analysis.

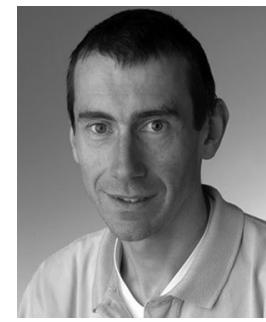

Konstantinos Papathanassiou (M'02-SM'03F'14) received the Dipl. Ing (Hons.) degree and the Dr. degree (Hons.) from the department of Physics, Technical University of Graz, Graz, Austria, in 1994 and 1999, respectively.

From 1992 to 1994 he was with the Institute for Digital Image Processing (DIBAG), Joanneum Research, Graz. Between 1995 and 1999 he worked with the Microwaves and Radar Institute (HR), German Aerospace Center (DLR), Wessling, Germany. From 1999 to 2000, he was an EU Postdoctoral Fellow with Applied Electromagnetics (AEL), St. Andrews, Scotland. Since October 2000 he has been with the Microwaves and Radar Institute (HR), German Aerospace Center (DLR). He is a Senior Scientist leading the Information Retrieval research group at DLR-HR. His main research interests include polarimetric and interferometric processing and calibration techniques, polarimetric SAR interferometry, and the quantitative parameter estimation from SAR data, as well as in SAR mission design and SAR mission performance analysis.

Mr. Papathanassiou is a member of DLR's TanDEM-X and Tandem-L science teams, JAXA's ALOS-2 Cal-Val teams, ESA's BIOMASS Mission Advisory Group, SAOCOM-SC Expert Team, and JAXA's Carbon and Kyoto Initiative and NASA's GEDI Mission Science Team. He was the recipient of the IEEE GRSS IGARSS Symposium Prize Paper Award in 1998, the Best Paper Award of the European SAR Conference (EUSAR) in 2002, the DLR science award in 2002, and the DLR's Senior Scientist Award in 2011. 